\title{
Barriers and facilitators experienced in collaborative prospective research in orthopaedic oncology
}

\author{
A QUALITATIVE STUDY
}

\section{J. S. Rendon, M. Swinton, N. Bernthal, M. Boffano, T. Damron, N. Evaniew, P. Ferguson, M. Galli Serra, W. Hettwer, P. McKay, B. Miller, L. Nystrom, W. Parizzia, P. Schneider, A. Spiguel, R. Vélez, K. Weiss, J. P. Zumárraga, M. Ghert}

McMaster University, Hamilton, Ontario, Canada

\section{Objectives}

As tumours of bone and soft tissue are rare, multicentre prospective collaboration is essential for meaningful research and evidence-based advances in patient care. The aim of this study was to identify barriers and facilitators encountered in large-scale collaborative research by orthopaedic oncological surgeons involved or interested in prospective multicentre collaboration.

\section{Methods}

All surgeons who were involved, or had expressed an interest, in the ongoing Prophylactic Antibiotic Regimens in Tumour Surgery (PARITY) trial were invited to participate in a focus group to discuss their experiences with collaborative research in this area. The discussion was digitally recorded, transcribed and anonymised. The transcript was analysed qualitatively, using an analytic approach which aims to organise the data in the language of the participants with little theoretical interpretation.

\section{Results}

The 13 surgeons who participated in the discussion represented orthopaedic oncology practices from seven countries (Argentina, Brazil, Italy, Spain, Denmark, United States and Canada). Four categories and associated themes emerged from the discussion: the need for collaboration in the field of orthopaedic oncology due to the rarity of the tumours and the need for high level evidence to guide treatment; motivational factors for participating in collaborative research including establishing proof of principle, learning opportunity, answering a relevant research question and being part of a collaborative research community; barriers to participation including funding, personal barriers, institutional barriers, trial barriers, and administrative barriers and facilitators for participation including institutional facilitators, leadership, authorship, trial set-up, and the support of centralised study coordination.

\section{Conclusions}

Orthopaedic surgeons involved in an ongoing international randomised controlled trial (RCT) were motivated by many factors to participate. There were a number of barriers to and facilitators for their participation. There was a collective sense of fatigue experienced in overcoming these barriers, which was mirrored by a strong collective sense of the importance of, and need for, collaborative research in this field. The experiences were described as essential educational first steps to advance collaborative studies in this area. Knowledge gained from this study will inform the development of future large-scale collaborative research projects in orthopaedic oncology.

Cite this article: Bone Joint Res 2017;6:307-314.

Keywords: Orthopaedic oncology, Collaborative research, Focus group

\section{Article focus}

- Multicentre prospective collaborative research is imperative to draw valid conclusions in orthopaedic oncology research due to the rarity of bone and soft-tissue sarcomas.

- The objective of this study was to identify barriers and facilitators encountered in large-scale collaborative research by orthopaedic oncologists currently involved, or interested, in prospective multicentre collaboration.

- Barriers and facilitators were identified through an in-person focus group in which participants discussed their experiences with collaborative research.

Bone Joint Res 2017;6:307-314. 


\section{Key messages}

- Four categories emerged from the discussion: the need for collaboration in the field; motivational factors for participating in collaborative research; barriers to participating in collaborative research; and facilitators for participating in collaborative research.

- Understanding the barriers and facilitators specific to orthopaedic oncologists is an essential educational first step for guiding collaborative studies in orthopaedic oncology.

\section{Strengths and limitations}

- The focus group represented a geographically diverse group of orthopaedic oncologists, with participants hailing from seven countries and three continents.

- The qualitative descriptive approach used minimises researcher bias as the method involves minimal interpretation of data and focuses on presenting and organising the data in the language used by participants.

- The unique research landscape within orthopaedic oncology may limit the external validity of the results.

\section{Introduction}

Bone and soft-tissue sarcomas represent less than $1 \%$ of all malignancies and are managed by multidisciplinary teams in specialty tertiary care centres. ${ }^{1}$ Although functional and survival outcomes improved significantly for these patients in the 1980 s due to advanced imaging techniques and aggressive chemotherapy protocols, the outcomes have only improved a little since that time. ${ }^{1}$ As with any rare disease, multicentre collaboration is critical to drive advances in evidence-based care.

As sarcomas are rare, individual orthopaedic oncological practices do not treat enough patients to conduct single-centre prospective comparative trials. Most research published by orthopaedic oncologists involves Level IV evidence with single-centre retrospective series. ${ }^{2}$ Prospective multicentre randomised controlled trials (RCTs) have not been successfully undertaken in orthopaedic oncology. Several factors affect this, including the accessibility of resources, recruitment, technological development and the expansion of research networks which are required to undertake clinical trials in surgery. ${ }^{3}$

The ongoing Prophylactic Antibiotic Regimens in Tumour Surgery (PARITY) trial is the first multicentre RCT to be conducted in orthopaedic oncology. ${ }^{4}$ It examines the comparative efficacy of long versus short postoperative antibiotic regimens in preventing infection after bone tumour surgery, ${ }^{5}$ and is currently enrolling patients in 37 sites in seven countries.

Given that there is now some experience of international collaboration in orthopaedic oncology, surgeons in this field have the opportunity to develop collaborative prospective studies. The aim of this study was to identify barriers to, and facilitators for, international collaborative research undertaken by orthopaedic oncologists involved in the PARITY Network in order to inform future similar initiatives.

Qualitative research currently has an important role. The methods used in this type of research are diverse and typically not familiar to the general audience. However, the capacity to evaluate and describe a given subject should be highlighted and favoured in areas where it is not possible to conduct research using conventional quantitative methods. Furthermore, the authors favour this form of research which could, with advantage, be used in large-scale studies, especially during their preliminary stages.

\section{Patients and Methods}

This qualitative study was conducted with orthopaedic oncologists involved in the PARITY Network in parallel with the annual Musculoskeletal Tumor Society Meeting in Orlando, Florida (October 2015). All members of the Network, including surgeons at active sites and those at sites which are not enrolling patients, were invited by email before the meeting to participate in a focus group discussion on their involvement. We used a convenience sample with the goal of using intensity sampling of the variable of experience with the PARITY Network. We approached all the surgeons already involved in or willing to participate in PARITY. An invitation was sent to 80 surgeons and research personnel. A total of 13 surgeons confirmed their voluntary participation in our study and attended the focus group discussion on 10 October 2015 in Orlando. The script for the focus group is available as supplementary material. The demographic information, which we collected from the participants included age, gender, ethnicity, country of practice, experience with research and level of involvement in the PARITY trial. The most important characteristics for eligibility were being an orthopaedic oncological surgeon and having expressed an interest in conducting collaborative, large-scale research in this field. The focus was not on age or gender, but on level of experience in RCTs, PARITY in particular.

This descriptive qualitative method of research is based on information collected from a pre-selected group of people. Participants are asked to disclose information in a neutral environment, where the researcher(s) have no power of manipulation of the information being provided. Purposeful sampling is a concept that has been explored within qualitative research and an advantage is that by incorporating information-rich cases it allows the thorough analysis of data and more relevant conclusions about a given subject.

The number of questions chosen for the focus group depended on such factors as the dimensions of the research question to be explored, the level of education of the participants, the anticipated level of comfort for discussion among participants and the amount of time scheduled for the discussion. We knew that the 
participants had a common experience (being involved to some extent in the PARITY network), most were familiar to each other, and all were highly educated. We were thus confident in our ability to cover a number of different areas related to their experience. We had also prioritised questions such that if there was insufficient time, we would have covered the most important topics. We had a generous amount of time (90 minutes) and, given the professional nature of participants, informed consent was quickly obtained which gave more time for discussion.

The research team, which included two orthopaedic surgeons (MG and NE), a resident in orthopaedic surgery (JR) and three research staff (including an experienced qualitative researcher; PS, PM and MS), developed and refined the questions. The questions were vetted within this team for clarity and face validity.

The participants in the focus group met at a single venue. The study was not conducted online or by teleconference. The discussion was digitally recorded, transcribed verbatim by an independent professional transcriptionist and anonymised by the person who led the discussion. Transcription occurred within two weeks and initial coding occurred within six weeks. The main analysis was then completed within two months and the writing extended over four months.

The study had ethical approval from the Hamilton Integrated Research Ethics Board (HiREB \#15-367).

Statistical analysis. We used a qualitative descriptive approach for analysis, a method that aims to provide a descriptive summary of the data, in the language of the participants, with minimal theoretical interpretation. ${ }^{6}$ Transcripts and field notes from the discussion were analysed using conventional qualitative analysis, with codes being derived directly from the data rather than using preconceived categories. ${ }^{7}$ Four investigators (SR, MS, MG and PS) independently undertook line-byline coding of the field notes and transcript, and met to develop the list of codes by consensus. They also assessed saturation of the data and agreed when saturation was achieved (i.e. when no new information was being provided). The research team reviewed the list of codes, discussed the relationships between them and organised them into categories, a process known as axial coding. ${ }^{8}$ The qualitative software programme NVivo, version 10.0 (QSR International, Doncaster, Australia) was used for data management and analysis. Quantitative demographic data were analysed using descriptive statistics.

\section{Results}

The 13 participants were male orthopaedic surgeons with a mean age of 41 years (34 to 54); nine (69\%) were Caucasian, three (23\%) were Hispanic/Latino and one (8\%) was of mixed ethnicity. They represented university affiliated or tertiary referral orthopaedic oncology
Table I. Participant demographics

\begin{tabular}{|c|c|}
\hline Participants $(n=13)$ & \\
\hline \multicolumn{2}{|l|}{ Gender (n) } \\
\hline Male & 13 \\
\hline Female & 0 \\
\hline \multicolumn{2}{|l|}{ Age (yrs) } \\
\hline Mean (range) & 40.5 (34 to 54$)$ \\
\hline \multicolumn{2}{|l|}{ Ethnicity } \\
\hline Caucasian & 9 \\
\hline Hispanic/Latino & 3 \\
\hline Aboriginal & 0 \\
\hline Asian & 0 \\
\hline Middle Eastern & 0 \\
\hline South-East Asian & 0 \\
\hline African & 0 \\
\hline Mixed & 1 \\
\hline Other & 90 \\
\hline \multicolumn{2}{|l|}{ Continent (Country) of practice } \\
\hline Europe (Denmark, Italy, Spain) & 3 \\
\hline North America (Canada/United States) & $7(1 / 6)$ \\
\hline South America (Argentina/Brazil) & $3(2 / 1)$ \\
\hline \multicolumn{2}{|l|}{ Occupation } \\
\hline Orthopaedic Surgeon & 13 \\
\hline \multicolumn{2}{|l|}{ Type of institution } \\
\hline University affiliated/tertiary referral & 13 \\
\hline Community affiliated (with physicians in training) & 0 \\
\hline Community affiliated (no physicians in training) & 0 \\
\hline \multicolumn{2}{|l|}{ Previous involvement in other research? } \\
\hline Yes/no & $13 / 0$ \\
\hline \multicolumn{2}{|l|}{ Type of other research } \\
\hline Observational & 13 \\
\hline Randomised controlled trials & 3 \\
\hline Qualitative & 5 \\
\hline Basic science & 9 \\
\hline Other & 2 \\
\hline \multicolumn{2}{|l|}{ Level of experience in the PARITY trial } \\
\hline Ready for enrolment & 6 \\
\hline \multicolumn{2}{|l|}{ Ready for enrolment, and already enrolled } \\
\hline At least 1 patient & 4 \\
\hline Trying to participate & 5 \\
\hline Not able to participate & 2 \\
\hline
\end{tabular}

practices in seven countries: Argentina, Brazil, Italy, Spain, Denmark, United States and Canada. All reported prior involvement in research, with three (23\%) involved with an RCT. They represented all stages of involvement in the PARITY Network, six with active participation, of whom four had already enrolled at least one patient. Five were trying to participate and two were unable to participate (Table I).

Four categories emerged from the discussion: the need for collaboration in this area; motivational factors for participating in collaborative research; barriers to participation and facilitators for participation (Table II and Fig. 1).

The need for collaborative research. All participants valued collaborative research and agreed that it is needed in orthopaedic oncology due to the rare nature of bone tumours:

"[t]here are just things that we cannot answer by ourselves given the nature of [the] disease." And: "We work on difficult problems; these are ultra-rare diseases and without collaboration, it's difficult for me to envisage how there's going to be meaningful numbers and clinical research to really drive the field forward". 
Table II. Summary of barriers to and facilitators for collaboration in orthopaedic oncological research

\begin{tabular}{|c|c|c|}
\hline Barriers & Facilitators & Oncology-specific \\
\hline Institutional: Lack of research staff/infrastructure & $\begin{array}{l}\text { Institutional: research infrastructure, institutional } \\
\text { research board, collegiality/support }\end{array}$ & $\begin{array}{l}\text { Barrier: lack of infrastructure (in orthopaedic } \\
\text { oncology divisions) Facilitator: collegiality }\end{array}$ \\
\hline $\begin{array}{l}\text { Personal-level challenges: limited bandwidth/capacity, } \\
\text { time-consumption, fatigue from fighting barriers }\end{array}$ & Shared nationality where the protocol originated & \\
\hline $\begin{array}{l}\text { Protocol-related: country regulations, relevance of } \\
\text { research question }\end{array}$ & Leadership of investigators & $\begin{array}{l}\text { Barrier: relevance of research question (infection) } \\
\text { in the oncology field }\end{array}$ \\
\hline $\begin{array}{l}\text { Documentation procedures and inter-country } \\
\text { transferability: paperwork, translation }\end{array}$ & Authorship in related publications & \\
\hline Funding & $\begin{array}{l}\text { Trial set-up: vetted, transparence, equipoise of } \\
\text { research question }\end{array}$ & $\begin{array}{l}\text { Facilitator: Transparent protocol design of PARITY } \\
R C T\end{array}$ \\
\hline
\end{tabular}

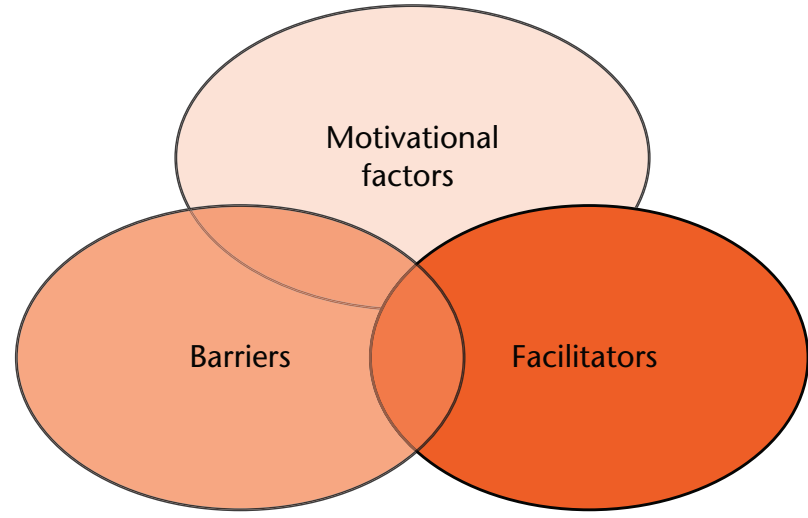

Fig. 1

Three themes emerged from the discussion: motivational factors for collaborative research; barriers to research; and facilitators for research.

Surgeons described how collaboration on multicentre trials is "long overdue - it's something that we've been talking about for years and years" and is necessary in order to generate evidence:

If you're a teaching hospital and your residents say, "Dr. $X$, why do you keep antibiotics on until the drain is out?" And my best answer is, "Shut up, kid, that's the way I do it." [group laughter] ...I'm supposed to be an academic physician - that's not okay.

One participant acknowledged that "evidence-based medicine is the future" while another commented on the lack of evidence-based research in the field:

I think that at this past meeting that $95 \%$ of the papers are retrospective studies. There was practically [no] randomised, controlled, multi-centre....this is what has to be done to really get evidence going in our field.

One surgeon explained: "We've heard a lot of information from retrospective stud[ies] and you can't base all of your decisions on retrospective studies."

Motivational factors for participating in collaborative research. The participants described various factors that motivated them to participate in collaborative research including proof of principle, relevance of the research question, the opportunity for learning how to do larger-scale research and wanting to be involved in a collaborative research community. As PARITY is the first RCT undertaken by orthopaedic oncologists, some discussed the need to establish that the orthopaedic oncological community can successfully complete an RCT as a reason for participating, "It's a really good study to demonstrate that this is actually possible on an international basis." And:

The PARITY trial is unprecedented. I think having a bunch of surgeons get together, involved in a truly randomised, controlled trial... it's bit of a proof of principle project...this is trailblazing and I think it's exciting for all of us who are involved.

One surgeon described how the relevance of the research question motivated him to participate and reflected on how it might motivate others to participate:

One of the best things for me, to get involved in this program...is the good question ... [infection is a] very important problem, that all of us, independent of the country we work in, in the hospital we work in, we have that problem...if you do musculoskeletal oncology, you get infections...if you get a good question to be answered, people would get involved.

A junior surgeon described how the opportunity to learn about the research process was a motivation for him:

I think, as one of the younger members of the collaboration, it's an extremely valuable learning opportunity just to see what goes into this process... just to know what goes into it and all the work and the time and barriers that there are.

A number of participants spoke of the desire to be part of a research community as motivation:

[A]fter a while, everybody's participating and there's almost this sense that you have to be a part of it, that this is something big and different and practice-changing going on and you really want yourself and your institution to be making a contribution.

Eventually [someone else is] going to come up with some idea that we're all going to want to trial. Or maybe I'll ask a basic science question that I think is suitable for the larger membership...how am I going to expect the membership to participate if I haven't voted with my feet? ...As we move forward, we're all going to have ideas that we want to see asked this way. So, I think it's important to support each 
other in this.

Barriers to participating in collaborative research. While participants highlighted the overwhelming need and motivation for participating in collaborative research, all described the many barriers to their participation, including those from sites with existing research infrastructure and prior research experience. Some spoke of the difficulties presented by involvement in collaborative research:

I never envisioned how difficult it would be to do this... Lawyers are involved; exchange of information; all that stuff gets into play...it sounds like, in a perfect world, it would be easy but it really is not.

One of the things that I've learned from this is that these types of studies are really hard...I can't think of a simpler question with a less toxic intervention that what we're doing right now and it has been incredibly difficult, I think, for everybody involved to participate... So, yes, it can be done but we really have to think about the questions we're asking and make sure that they're going to be important if we want to be successful.

There was a collective sense of fatigue expressed by surgeons, and an articulated sense of needing to "fight" to overcome barriers and participate in this kind of research. One participant explained that: "There's always more roadblocks, you figure you're trying to do something for a good cause, you know, decrease rates of infection and it all makes sense but you just keep hitting blocks along the way. It gets a little tiring after a while."

The barriers which were discussed were classified into the following: funding; personal barriers; institutional barriers; trial barriers and administrative barriers. Limited funding was identified by several surgeons as a barrier to their participation. One explained that:

The financial limitations - it probably all goes back to that...... if something's going to make you money, usually you can find people to help you do it so it's difficult to advocate something just on scientific merit, especially if in our institution being in PARITY actually costs us money rather than not even bringing in money.

[Our institution] just create[s] new barriers so we cannot go through the process. There's some legal stuff and they just said that we cannot get money from elsewhere in our country but they don't want to give us money...

Personal barriers were related to demanding workloads and limited time:

For most people that I've been talking to about this trial [they] would maybe, in principle, very much like to participate but fear that they are already so much drowning in work and don't have local finance and research support that it's really very difficult to envision that, with all the work that you already have to take responsibility for that you can take on another responsibility to contribute on a reasonable basis.
The staff is supportive of all this but it's been more time consuming than I had imagined. I haven't had the time to dedicate to it but I think the amount of time that it needs.

Institutional barriers included a lack of research staff and infrastructure, and a lack of recognition. Some surgeons specifically identified the lack of research staff:

I was kind of smiling when I looked at the registration form specifying the number of staff surgeons that we've got in our department, which are eight...And the number of allocated research personnel is zero... that I think is the most prominent barrier for us.

Frankly, most people don't have the research support except at specific institutions to do it and you really need to have a study coordinator, at the very minimum to do these sorts of studies.

Trial barriers included the lack of relevance of the protocol to their practices (related to the type of antibiotic) and the lack of enthusiasm from interdisciplinary partners. One surgeon explained how the country in which he works uses a different antibiotic, and how that in turn influences the relevance of the research question:

PARITY approached this by trying to simplify the question and really get two homogeneous groups but then that goes into detriment, especially with European centres, where for example, the findings of Cefazolin being two grams post-op, that's not what we're going to do... So for us, as European centres, it's going to answer a worldwide question about length of antibiotics but not about our own antibiotic or current clinical practice.

A number of surgeons described how a barrier exists when the research question doesn't resonate with other disciplines:

The ID guys might get excited that some long term benefit is there if we kind of understand our usage of antibiotics better but folks who are used to seeing oncologic studies with really clear outcomes that you're trialing a new drug, I mean, just trying to pitch them that this is an important topic was really hard.

It's an awkward position to be in because orthopaedic departments generally don't know how to run randomised clinical trials so the support staff is not within your department. And then the cancer centres don't understand what you're doing anyway. It's kind of hard to advocate that this is actually a cancer study.

Administrative barriers included the large amount of paperwork related to participating in an RCT and, for some participants, the time involved in translating the trial documents into the language spoken in their country.

Facilitators for participating in collaborative research. Facilitating factors for participating in research were organised into the following: leadership; institutional facilitators; authorship; trial set-up; and Methods Centre 
support. Leadership at different levels was seen as a facilitator. One surgeon described how the support of leadership from professional societies influenced his participation in the PARITY trial:

When we hear our Society leadership at the podium every year talking about [PARITY] and how important it is and how exciting it is - it's hard not to buy into that and I think having the leadership buy in was certainly a factor for me in trying to implement this in our institution.

A junior surgeon shared how the support of the fellowship directors had a positive influence:

The other part, as a younger guy going through is seeing a lot of the Fellowship directors jumping on board and being really enthusiastic about PARITY ....you sort of see these people as the leaders and the teachers in the field and it seems meeting after meeting, they're involved, they're supportive of it....it's a really nice way to kind of pyramid down from [the Principal Investigator for the trial] to the Fellowship directors to the younger members of the Society.

Many spoke highly of the leadership shown by the Principal Investigator for the PARITY trial and how it is a facilitator for collaborative research: "[Her] enthusiasm is infectious for this, there's no question...I think that [her] enthusiasm and her drive and passion for this is a huge enabling factor in seeing its success."

Institutional facilitators included research infrastructure, industry to subsidise funding from an investigatorinitiated trial, and supportive colleagues. One surgeon described how the research infrastructure enabled his site to participate:

For our division of orthopaedics there are six surgeons - two of us, oncology surgeons... and we've got three and a half full time clinical research staff and two and a half of those dedicated to oncology so I will admit, freely admit, that is a huge enabling factor for us that permitted us to participate right off the bat.

Another participant explained how his site did not have a lot of infrastructure, but used funding from industry to offset the financial losses resulting from participating in investigator-initiated studies:

At our institution, there's a long track record for people doing industry studies. And because of that, we've been able to support clinical research coordinators within the department... I've been able to do a few industry-based studies that support my research coordinator and that has basically funded her to do trials like this where we're not really going to make any money on it.

Supportive colleagues were identified as facilitators by some participants. One described how having an advocate at his institution facilitated his institution's involvement with PARITY:

I have a colleague who's a medical oncologist who starts a lot of clinical trials and brings a lot of money into the department...he understands the importance of our institution being a part of a big effort like this. That it would be good in the long run for our institution even though there's no financial compensation and he actually championed that and said "Look guys, I know we're losing money but we have to do this and we're doing it."

Authorship was identified as a facilitator for participation at both the individual and at trial level:

I'd be lying if I said authorship didn't matter. It does. Most of us are in academic centres. The currency that I deal in is with publications...participating in studies like this adds gravitas to my list of publications so from a completely selfish standpoint, it is helpful.

Authorship is really helpful and also gives a confirmation of the validity of the process and the validity of the trial.

The process of how the PARITY trial was set up and presented to surgeons was also identified as facilitator:

I think a lot of the reason that PARITY, specifically, was successful is that it was well set up and vetted before it started.

The setup process was transparent in that the people who were eventually going to be participants saw the evolution of the question.

This is a question that, I think, [the Principal Investigator for the PARITY] demonstrated that there's equipoise for. If you go through that process, then you realize we don't know what the right answer is and if you buy into that, then you can enrol patients.

Support from the PARITY Methods Centre was described as a "fundamental" facilitator by offering assistance with the legal and regulatory processes related to trial participation. One surgeon explained that, "to have a consistent contact is certainly helpful, and I would say, critical, in a situation like this."

\section{Discussion}

This qualitative study is based on a discussion of a group of surgeons involved in a specific RCT (PARITY), the first in orthopaedic oncology. We applied the concepts of descriptive qualitative research, and designed and executed a study without manipulating the information provided by participants. Purposeful sampling is a key element in this form of research which involves collecting information from participants with an interest in a given area of study. ${ }^{9}$ This involved surgeons enrolled in or willing to be part of PARITY, who had a wide range of experiences and perspectives with regard to participation in the RCT. Another key concept relies on participants meeting in a non-artificial setting, thus providing raw and valid information that can be extrapolated to other similar scenarios. Qualitative sampling takes into account not only the characteristics of the participants, but also their 
context, ${ }^{10}$ which in principle contributes to the diversification of the information, which is gathered and incorporated into the presentation of data.

We aimed to identify barriers to, and facilitators for, participation in collaborative research in musculoskeletal oncology. The discussion provided detailed information about the experiences of collaborating in a large-scale study. A shared feeling of fatigue was expressed about barriers which were frequently experienced as external factors that influenced the ability of surgeons to participate in the PARITY trial. At the same time, the participants voiced their appreciation and motivation for ongoing and future collaborative research based on their experiences with the trial. PARITY is a drug trial in orthopaedic oncology. The problems found are therefore not inherent in its design but depend on the expertise of orthopaedic surgeons, their research staff, and their centres in this type of study.

The challenges of conducting RCTs have been known for many decades. ${ }^{11-17}$ There has been a constant increase in the number and complexity of institutional and national barriers as medical innovation has evolved. Cook ${ }^{11}$ emphasised the importance of collaboration among surgical research communities when overcoming barriers and pointed to collaboration as a key component of a well designed trial. Our focus group similarly expressed strong support for collaboration as a key motivator in the PARITY trial.

Rendell et al ${ }^{18}$ analysed the incentives and disincentives to participation by clinicians in RCTs in a systematic review of the literature. They identified several incentives to collaboration and recruitment of patients such as the participation of academic-research groups, the availability of research-support staff, interest in research and the surgeon's level of comfort when explaining trials to patients. Although our focus group did not touch upon issues of recruitment, we found that surgeons similarly are motivated by involvement in academic collaboration and consider the availability of research and support staff to be important facilitators for collaborative research.

Søreide et $\mathrm{al}^{3}$ provided a narrative review of the topic, describing the associated challenges and recent evidence of a transcontinental collaborative research network in the field of surgery and different subspecialties. The challenges which were discussed resonate with our findings, including the challenges related to: lack of financial support and difficulties experienced with investing time in endeavours that are usually not reimbursed; difficulty obtaining formal translations of consent forms and administrative documents; and dealing with discrepancies of legislation and regulations specific to each country. ${ }^{3}$ However, the surgeons in our focus group had similar opinions about the importance of adopting a culture of leadership and the identification of research centres with the appropriate intellectual and physical infrastructure to ensure widespread ongoing collaboration.
Similar qualitative studies have been conducted in various specialties such as cancer, primary care, infection, mental health and community care. ${ }^{19-21}$ They have helped in identifying and understanding problems relating to RCTs such as the investment of time required in organisation and governance procedures. Based on our findings, the field of orthopaedic oncology appears to share similar barriers with those of prospective collaborative research. Although this is the first qualitative study of which we are aware in orthopaedic oncology with a focus on RCTs, other surgical studies have used qualitative methods to identify team-related factors and the need for shared leadership as important determinants in the success of RCTs. ${ }^{22}$

The consolidation and constant growth of a research network is essential in order to improve both opportunities for, and the quality of, collaborative surgical research. Leadership in surgical research should be emphasised as an important prerequisite to success. Surgeons with experience in methodology who have access to international research networks can overcome many obstacles of conducting RCTs, while ensuring the production of high quality research and maintaining access to funding. ${ }^{12,23}$ Our focus group confirmed the critical importance of leadership and support for research for the success of international collaboration.

Audrey ${ }^{24}$ conducted focus group discussions with participants and non-participants in the early stages of two large international cancer clinical trials and reported that they were crucial to adjustments of the study protocol that ensured the success of the trials. This work supports the value of qualitative research in evidence-based medicine as a beneficial strategy to all aspects of collaboration, especially at the early stages of planning an RCT. The authors and participants in our study valued the experience of the focus group as an effective strategy to maintain and further develop a collaborative network in the field of orthopaedic oncology. Thus, our experience was similar to that reported by Audrey, in that early qualitative work can inform collaborative efforts, particularly those of international scale.

Strengths and limitations. Musculoskeletal tumours are rare and often described as 'orphan' diseases. The strength of this study is that, to date, orthopaedic oncologists have not explored these areas of qualitative research. This study also might be used to investigate research into other rare diseases, in which there may be similar challenges in conducting RCTs. Moreover, this study's infrastructure is based on PARITY, which is, for the first time, capturing and exploring the experiences of orthopaedic oncological surgeons in different stages of involvement in RCTs. It therefore represents a step forward for future large-scale research in orthopaedic oncology and other 'orphan' conditions.

The information for the study was collected from a focus group discussion. The group was multinational 
and, given the qualitative nature of the research, this was beneficial and enriched the information obtained. However, a limitation of this study is that some international investigators may not have been able to attend the discussion for logistical reasons of time and cost of travel to the United States. Nevertheless, the discussion eliminates the challenges encountered in multicentre studies by relying on information collected at a single time, without the requirement for continued participation and contribution.

Although important challenges to collaboration have been encountered though this qualitative research study, most have been overcome thanks to a growing interest in maintaining a solid collaborative network, which strives to find answers of common benefit to orthopaedic oncologists. The focus group strategy implemented, using PARITY as the means to answer our research question, is therefore an example of how a scientific society can produce and share knowledge with the goal of overcoming challenges and consolidating scientific efforts to facilitate future large-scale research studies.

\section{References}

1. Ottaviani G, Jaffe N. The Epidemiology of Osteosarcoma. In: Jaffe N, Bruland SO, Bielack S, eds. Pediatric and Adolescent Osteosarcoma. Boston, Springer US, 2010:3-13.

2. Evaniew N, Nuttall J, Farrokhyar F, Bhandari M, Ghert $\mathbf{M}$. What are the levels of evidence on which we base decisions for surgical management of lower extremity bone tumors? Clin Orthop Relat Res 2014;472:8-15.

3. Søreide K, Alderson D, Bergenfelz A, et al. Strategies to improve clinical research in surgery through international collaboration. Lancet 2013;382:1140-1151.

4. Ghert M, Deheshi B, Holt G, et al. Prophylactic antibiotic regimens in tumour surgery (PARITY): protocol for a multicentre randomised controlled study. BMJ Open 2012;2:e002197.

5. PARITY Investigators. Prophylactic antibiotic regimens in tumour surgery (PARITY): a pilot multicentre randomised controlled trial. Bone Joint Res 2015;4:154-162.

6. Sandelowski M. Whatever happened to qualitative description? Res Nurs Health 2000;23:334-340.

7. Hsieh HF, Shannon SE. Three approaches to qualitative content analysis. Oual Health Res 2005;15:1277-1288.

8. Strauss A, Corbin J. Basics of qualitative research. Vol. 15. Newbury Park, Sage, 1990.

9. Patton Mo. Qualitative evaluation and research methods. Beverly Hills, Sage, 1990:169-186.

10. Marshall MN. Sampling for qualitative research. Fam Pract 1996;13:522-525.

11. Cook JA. The challenges faced in the design, conduct and analysis of surgical randomised controlled trials. Trials 2009;10:9.

12. Garas G, Ibrahim A, Ashrafian H, et al. Evidence-based surgery: barriers, solutions, and the role of evidence synthesis. World J Surg 2012;36:1723-1731.

13. Haines SJ. Randomized clinical trials in the evaluation of surgical innovation. $J$ Neurosurg 1979;51:5-11.

14. Lawrence W Jr. Some problems with clinical trials. James Ewing lecture. Arch Surg 1991;126:370-378.

15. McLeod RS. Issues in surgical randomized controlled trials. World J Surg 1999;23:1210-1214.

16. Pollock AV. The rise and fall of the random controlled trial in surgery. Theor Surg 1989;4:163-170

17. van der Linden W. Pitfalls in randomized surgical trials. Surgery 1980;87:258-262.

18. Rendell JM, Merritt RD, Geddes JR. Incentives and disincentives to participation by clinicians in randomised controlled trials. Cochrane Database Syst Rev 2007:2:21.

19. de Salis I, Tomlin Z, Toerien M, Donovan J. Using qualitative research methods to improve recruitment to randomized controlled trials: the Quartet study. J Health Serv Res Policy 2008;13(Suppl 3):92-96.
20. Donovan JL, Paramasivan S, de Salis I, Toerien M. Clear obstacles and hidden challenges: understanding recruiter perspectives in six pragmatic randomised controlled trials. Trials 2014;15:5.

21. Donovan JL, Rooshenas $\mathbf{L}$, Jepson $\mathbf{M}$, et al. Optimising recruitment and informed consent in randomised controlled trials: the development and implementation of the Quintet Recruitment Intervention (QRI). Trials 2016;17:283.

22. Strong S, Paramasivan S, Mills N, et al. 'The trial is owned by the team, not by an individual': a qualitative study exploring the role of teamwork in recruitment to randomised controlled trials in surgical oncology. Trials 2016;17:212.

23. Jarman AF, Wray NP, Wenner DM, Ashton CM. Trials and tribulations: the professional development of surgical trialists. Am J Surg 2012;204:339-346.

24. Audrey S. Qualitative research in evidence-based medicine: improving decisionmaking and participation in randomized controlled trials of cancer treatments. Palliat Med 2011;25:758-765.

Funding Statement

None declared

Author Contribution
J. S. Rendon: Study design, data acquisition, data analysis, manuscript preparation and editing.

M. Swinton: Study conception, study design, data acquisition, data analysis, manuscript preparation and editing.

N. Bernthal: Data acquisition, manuscript editing and review.

M. Boffano: Data acquisition, manuscript editing and review.

T. Damron: Data acquisition, manuscript editing and review.

N. Evaniew: Study design, manuscript editing and review.

P. Ferguson: Data acquisition, manuscript editing and review.

M. Galli Serra: Data acquisition, manuscript editing and review.

W. Hettwer: Data acquisition, manuscript editing and review.

P. McKay: Study design, manuscript editing and review.

B. Miller: Data acquisition, manuscript editing and review.

L. Nystrom: Data acquisition, manuscript editing and review.

W. Parizzia: Data acquisition, manuscript editing and review.

P. Schneider: Study design, data analysis, manuscript preparation and editing.

A. Spiguel: Data acquisition, manuscript editing and review.

R. Vélez: Data acquisition, manuscript editing and review.

K. Weiss: Data acquisition, manuscript editing and review.

J. P. Zumárraga: Data acquisition, manuscript editing and review.

ICMJE Conflicts of Interest

- None declared

(c) 2017 Ghert et al. This is an open-access article distributed under the terms of the Creative Commons Attributions licence (CC-BY-NC), which permits unrestricted use, distribution, and reproduction in any medium, but not for commercial gain, provided the original author and source are credited.

Author Affiliation

J. S. Rendon, MD,

M. Swinton, MSc,

N. Evaniew, MD, PhD

- P. McKay, BSC,

P. Schneider, BSc, McMaster University, 293 Wellington Street North, Suite 110,

Hamilton, ON, Canada.

N. Bernthal, MD, Orthopaedic Surgeon, assistant Professor, Department of

Orthopaedic Surgery, University of California Los Angeles, $125016^{\text {th }}$ Street, Suite 3142, Santa Monica, Los Angeles, CA, 90404, USA

M. Boffano, MD, Orthopaedic Surgeon, Department of Orthopaedics, AO Città della Salute e della Scienza di Torino, Via Zuretti 29, Torino, 10126 Italy.

- T. Damron, MD, Orthopaedic Surgeon, Professor, State University of New York (SUNY) Upstate Medical University, 6620 Fly Road, Suite 100, East Syracuse, NY, 13057, USA.

- P. Ferguson, MD, MSc, Orthopaedic Surgeon, Division Chair, Division of Orthopaedic Surgery, University of Toronto, 600 University Avenue, Suite 476G,

M. Galli Serra, MD

W. Parizzia, MD, Universidad Austral, Av. Juan Domingo Péron 1500, 4to. Piso,

Derqui B16290DT Pilar, Buenos Aires, Argentina.

W. Hettwer, MD, Orthopaedic Surgeon, Department of Orthopaedic Surgery,

W. Hettwer, MD, Orthopaedic Surgeon, Department of Or
University of Copenhagen, Copenhagen, 2100, Denmark

University of Copenhagen, Copenhagen, 2100, Denmark.
B. Miller, MD, MS, Orthopaedic Surgeon, Assistant Professor, Department of B. Miller, MD, MS, Orthopaedic Surgeon, Assistant Professor, Department of
Orthopaedics and Rehabilitation, University of lowa, 200 Hawkins Drive, 01015 JPP, lowa City, USA.

- L. Nystrom, MD, Orthopaedic Surgeon, Assistant Professor, Department of Orthopaedic Surgery and Rehabilitation, Loyola University Chicago, 2160 South First Avenue, Maywood, IL, 60153, USA.

- A. Spiguel, MD, Orthopaedic Surgeon, Assistant Professor, Department of Orthopaedics and Rehabilitation, University of Florida, 3450 Hull Road, Gainesville, FL, 32607, USA.

- R. Vélez, MD, PhD, Orthopaedic Surgeon, Associate Professor, Department of Orthopaedic Surgery and Traumatology, Hospital Vall d'Hebron, Pg. Vall d'Hebron 119-129, 2a planta, Barcelona, Spain.

K. Weiss, MD, Orthopaedic Surgeon, Assistant Professor, Department of Orthopaedic Surgery, University of Pittsburgh, 5200 Centre Avenue, Shadyside Medical Building, Suite 415, Pittsburgh, PA, USA.

- J. P. Zumárraga, MD, MSc, Orthopaedic Surgeon, Department of Orthopaedics and Traumatology, Universidade de São Paulo, Universidade de São Paulo, Rua Dr. Ovídio Pires de Campos, 333, Cerqueira Cesar, São Paulo, SP, Brazil.

M. Ghert, MD, FRCSC, Orthopaedic Surgeon, Associate Professor, Department of M. Ghert, MD, FRCSC, Orthopaedic Surgeon, Associate Professor, Department of
Surgery, McMaster University, 711 Concession Street, Surgical Offices B3 169A | Hamilton, ON, Canada. 